\title{
Evaluation of three commercial metal artifact reduction methods for CT simulations in radiation therapy
}

\author{
Jessie Y Huang ${ }^{1,2}$, James R Kerns ${ }^{1,2}$, Jessica L Nute2,3, Xinming Liu' ${ }^{2,3}$, Francesco C Stingo ${ }^{2,4}$, \\ David S Followill ${ }^{1,2}$, Dragan Mirkovic ${ }^{1,2}$, Rebecca M Howell ${ }^{1,2}$, Stephen F Kry ${ }^{1,2}$ \\ ${ }^{1}$ Department of Radiation Physics, The University of Texas MD Anderson Cancer Center, Houston, TX, USA. \\ ${ }^{2}$ The University of Texas Health Science Center Houston, Graduate School of Biomedical Sciences, Houston, TX, USA. \\ ${ }^{3}$ Department of Imaging Physics, The University of Texas MD Anderson Cancer Center, TX, USA. \\ ${ }^{4}$ Department of Biostatistics, The University of Texas MD Anderson Cancer Center, Houston, Texas, USA.
}

Received March 19, 2014; Published Online April 08, 2014

[Presented at the Young Investigator's Symposium at the 2014 Annual Meeting of Southwest Chapter of American Association of Physicists in Medicine (AAPM) in San Antonio, Texas, USA]

\section{Conference Proceeding}

\begin{abstract}
Purpose: To evaluate the success of three commercial metal artifact reduction methods (MAR) in the context of radiation therapy treatment planning.

Methods: Three MAR strategies were evaluated: Philips O-MAR, monochromatic imaging using Gemstone Spectral Imaging (GSI) dual energy CT, and monochromatic imaging with metal artifact reduction software (GSI-MARs). The Gammex RMI 467 tissue characterization phantom with several metal rods and two anthropomorphic phantoms (pelvic phantom with hip prosthesis and head phantom with dental fillings), were scanned with and without metals (baseline). Each MAR method was evaluated based on CT number accuracy, metal size accuracy, and reduction in the severity of streak artifacts. CT number difference maps between the baseline and metal scan images were calculated, and the severity of streak artifacts was quantified using the percentage of pixels with > $40 \mathrm{HU}$ error ("bad pixels").
\end{abstract}

Results: Philips O-MAR generally reduced HU errors in the RMI phantom. However, increased errors and induced artifacts were observed for lung materials. GSI monochromatic 70keV images generally showed similar HU errors as con-

Presenting author: Jessie Y Huang; Department of Radiation Physics, The University of Texas MD Anderson Cancer Center, Houston, TX, USA.

Cite this article as:

Huang JY, Kerns JR, Nute JL, Liu X, Stingo FC, Followill DS, Mirkovic D, Howell RM, Kry SF. Evaluation of three commercial metal artifact reduction methods for CT simulations in radiation therapy. Int J Cancer Ther Oncol 2014; 2(2):020224.

DOI: $10.14319 /$ ijcto.0202.24 ventional $120 \mathrm{kVp}$ imaging, while $140 \mathrm{keV}$ images reduced HU errors. All the imaging techniques represented the diameter of a stainless steel rod to within $\pm 1.6 \mathrm{~mm}$ ( 2 pixels). For the hip prosthesis, O-MAR reduced the average \% bad pixels from $47 \%$ to $32 \%$. For GSI $140 \mathrm{keV}$ imaging, the $\%$ bad pixels was reduced from $37 \%$ to $29 \%$ compared to $120 \mathrm{kVp}$ imaging, and GSI-MARs further reduced it to $12 \%$. For the head phantom, none of the MAR methods was particularly successful.

Conclusion: O-MAR resulted in consistent artifact reduction but exhibited induced artifacts for metals located near lung tissue. GSI imaging at $140 \mathrm{keV}$ gave consistent reduction in HU errors and severity of artifacts. GSI-MARs at $140 \mathrm{keV}$ was the most successful MAR method for the hip prosthesis but exhibited induced artifacts at the edges of metals in some cases.

Innovation/Impact: CT streak artifacts caused by metal implants negatively affected the treatment planning process by 1 ) making it difficult to confidently contour the tumor and surrounding organs and 2) causing CT number errors that lead to dose calculation errors. In this study, we performed a comprehensive evaluation of these metal artifact reduction (MAR) techniques, using two types of metal implants that are commonly encountered in radiation therapy (a hip prosthesis and dental fillings) and metrics that are relevant for treatment planning and dose calculation accuracy (CT number accuracy, metal size accuracy, and severity of streak artifacts). 
Introduction/Background: The Philips O-MAR algorithm uses a projection modification approach for conventional polyenergetic CT imaging, while the Gemstone Spectral Imaging (GSI) system uses dual energy
CT data to generate monochromatic images to reduce beam hardening artifacts. The GSI-MARs algorithm further reduces artifacts by addressing the photon starvation aspect of metal streak artifacts.

Key Results:
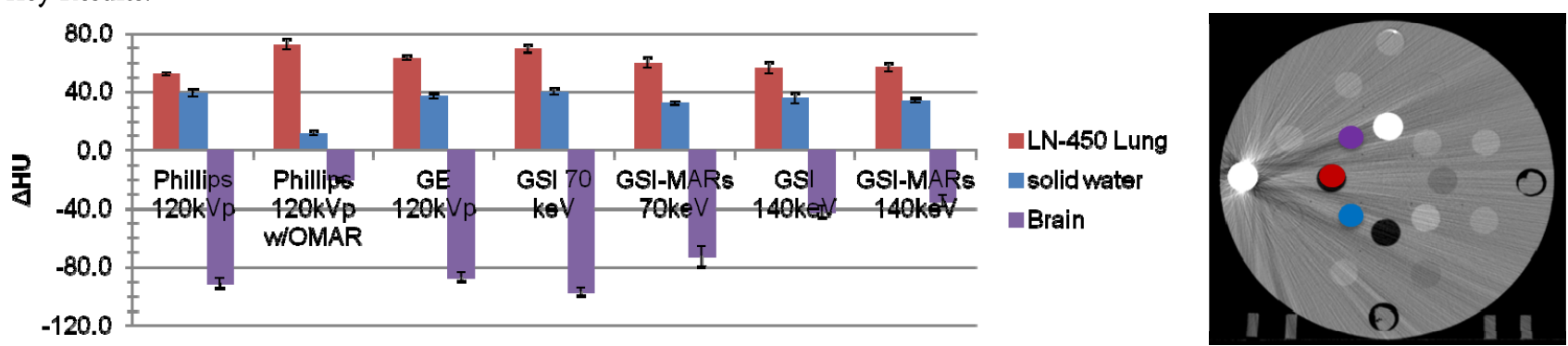

FIG. 1: Mean HU difference ( $\Delta \mathrm{HU}$ ) between baseline (no metal) and metal (stainless steel) scans of the RMI phantom. $\Delta$ HU was calculated based on the mean CT number for $14 \mathrm{~mm}$ diameter regions of interest. Error bars indicate the standard error of the mean for three repeated scans.
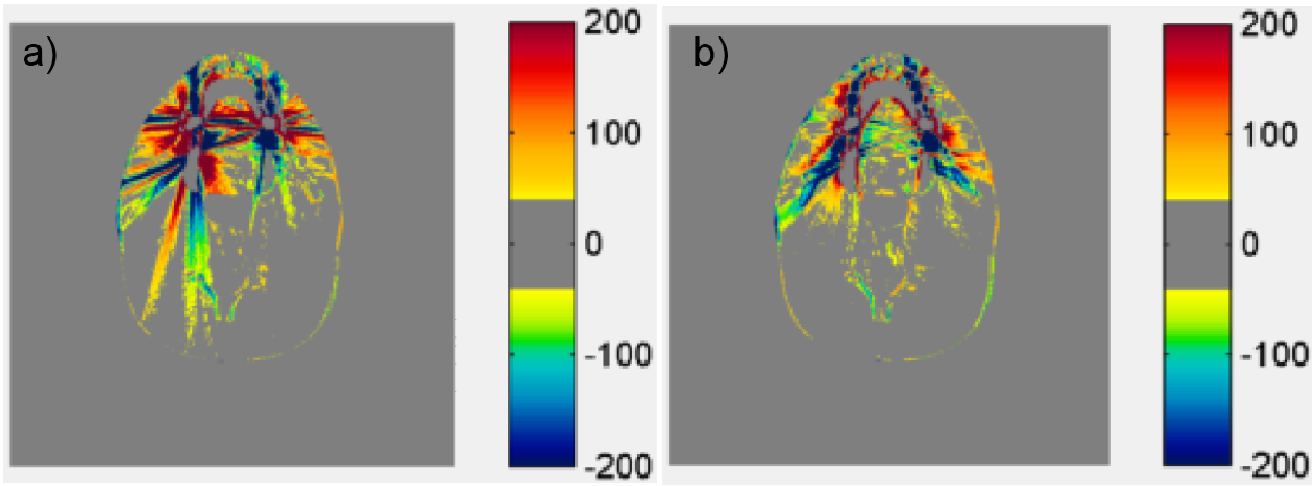

FIG. 2: CT number difference maps between baseline and metal scans for the head phantom with dental fillings for a) Philips $120 \mathrm{kVp}$ and b) Philips 120kVp with O-MAR. Regions of metal and air are excluded.
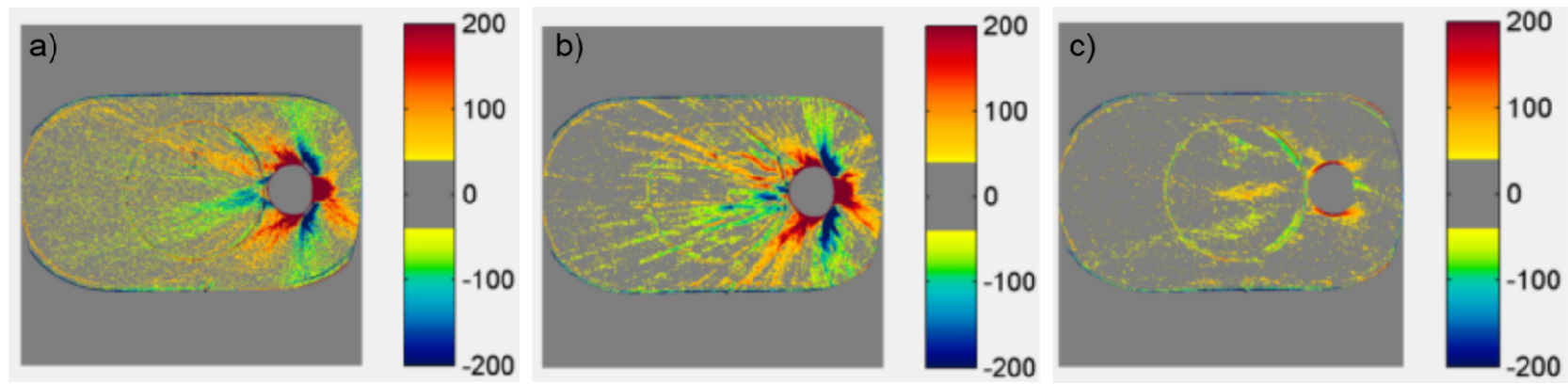

FIG. 3: CT number difference maps between baseline and metal scans for the pelvic phantom with hip prosthesis for a) GE 120kVp, b) GSI $140 \mathrm{keV}$, and GSI-MARs 140keV. Regions of metal and air are excluded. 